\title{
ORIGINAL ARTICLE Gene expression associated with suicide attempts in US veterans
}

\author{
JD Flory ${ }^{1,2,7}$, D Donohue ${ }^{3,7}$, S Muhie ${ }^{3}$, R Yang ${ }^{4}$, SA Miller ${ }^{3}$, R Hammamieh ${ }^{5}$, K Ryberg ${ }^{1}$ and R Yehuda ${ }^{1,2,6}$
}

According to a recent report from the Office of Suicide Prevention in the US Department of Veterans Affairs, veterans represent $8.5 \%$ of the US population, but account for $18 \%$ of all deaths from suicide. The aim of this study of psychiatric patients $(n=39 ; 87 \%$ male) was to compare blood gene expression data from veterans with a history of one or more suicide attempts to veterans who had never attempted suicide. The attempter and non-attempter groups were matched for age and race/ethnicity, and both groups included veterans with a diverse psychiatric history that included posttraumatic stress disorder (PTSD) and substance-use disorders. Veterans were interviewed for lifetime psychiatric history, including a detailed assessment of prior suicide attempts and provided a blood sample. Results of Ingenuity Pathway Analysis (IPA) identified several pathways associated with suicide attempts, including the mammalian target of rapamycin (mTOR) and WNT signaling pathways. These pathways are of particular interest, given their role in explaining pharmacological treatments for suicidal behavior, including the use of ketamine and lithium. These results suggest that findings observed in civilians are also relevant for veterans and provide a context for interpreting results observed in postmortem samples. In conclusion, an emerging body of work that shows consistency in findings across blood and brain samples suggests that it might be possible to identify molecular predictors of suicide attempts.

Translational Psychiatry (2017) 7, e1226; doi:10.1038/tp.2017.179; published online 5 September 2017

\section{INTRODUCTION}

Substantial progress has been made in identifying stable traits associated with suicidal behavior and concomitant biological markers, but there are no replicated clinical or biological predictors that distinguish acute or imminent risk from stable-risk traits. ${ }^{1}$ Much of the work that has been conducted to identify potential suicide biomarkers has been conducted by examining post-mortem brain tissue. ${ }^{2}$ Molecular markers or processes identified in post-mortem tissue may reflect long-standing risk and/or a more proximal precursor of the death from suicide. An unanswered question is whether the results obtained in postmortem tissue generalize to blood samples obtained from people who attempted, but did not complete suicide. If the same molecules or processes are observed in blood from people who did not die from suicide, this suggests an association with a more stable trait of risk. In accord with this view, recent research on suicide risk has increasingly focused on identifying molecular processes and networks in blood. ${ }^{3-6}$

Nearly one out of five people in the United States who die from suicide have served in the military, ${ }^{7}$ which indicates that this is a large proportion of the US population that requires monitoring and intervention for suicidal risk. Recent research on active duty service personnel and veterans has generally highlighted the importance of a diverse range of psychiatric disorders as contributing factors for attempts and suicide completion, including panic disorder and posttraumatic stress disorder (PTSD) ${ }^{8}$ as well as psychiatric problems that may have developed during military service such as depression, bipolar disorder, alcohol use disorder ${ }^{9}$ and intermittent explosive disorder. ${ }^{8}$ However, because suicide attempts occur in the context of many different psychiatric diagnoses and are independent of any single diagnosis, ${ }^{10}$ it is important to identify factors associated with suicide versus underlying psychiatric symptoms. That is, many studies of suicide that examine molecular markers and processes are conducted comparing people in relatively discrete diagnostic groups (for example, people with mood disorder or psychosis) to psychiatrically healthy controls (although see Niculescu et al. ${ }^{5}$ who studied a sample of men with a range of mood and psychotic disorders). In the case of post-mortem tissue, a typical comparison group consists of people who died from accidents without underlying psychiatric symptomatology. Such comparisons make it difficult to determine whether the observed biological signal is specific to suicide per se, or whether it reflects the complex psychiatric background, both of which are present in suicide attempts and deaths.

The aim of the current study was to identify molecular pathways and networks associated with suicide attempts using genomewide gene expression data obtained from peripheral blood samples of veterans. To minimize sources of heterogeneity that would make it difficult to detect molecular pathways in a relatively small sample, the study was conducted in veterans who had attempted suicide compared with veterans who were comparable by gender, race, ethnicity, age and psychiatric history but who had never made a suicide attempt. A psychiatric comparison group was chosen to maximize the clinical utility of the study as clinical providers are often faced with the dilemma of distinguishing

\footnotetext{
${ }^{1}$ Office of Mental Health, James J. Peters Veterans Affairs Medical Center, Bronx, NY, USA; ${ }^{2}$ Department of Psychiatry, Icahn School of Medicine at Mount Sinai, New York, NY, USA; ${ }^{3}$ The Geneva Foundation, Fort Detrick, MD, USA; ${ }^{4}$ Advanced Biomedical Computing Center at the Frederick National Labs for Cancer Research, Frederick, MD, USA; ${ }^{5}$ Integrative Systems Biology, US Army Medical Research and Material Command, USACEHR, Fort Detrick, MD, USA and ${ }^{6}$ Department of Neuroscience, Icahn School of Medicine at Mount

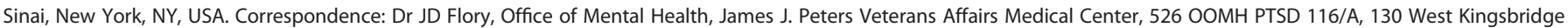
Road, Bronx, NY 10468, USA.
}

E-mail: Janine.Flory@mssm.edu

${ }^{7}$ These authors contributed equally to this work.

Received 25 October 2016; revised 5 May 2017; accepted 9 May 2017 
between patients who will self-harm and patients who will not. In addition, the psychiatric comparison group allows for clearer interpretation as any observed biological differences would reflect suicide history rather than the confounding influence of psychopathology and associated features (for example, medication use).

\section{MATERIALS AND METHODS}

\section{Participants}

One hundred and thirty-one veterans (61 who had made one or more suicide attempts and 70 who had never made a suicide attempt) provided blood samples and completed a clinical interview. Patients were referred to the study by mental health providers at the James J Peters VAMC or were self-referred. All study procedures, including recruitment from the inpatient psychiatry unit, were approved by the Institutional Review Boards at the James J Peters VAMC, the Icahn School of Medicine at Mount Sinai and the US Army Medical Research and Materiel Command Human Research Protection Office; all study procedures, including screening and evaluation, were conducted after the veterans provided written, informed consent.

\section{Clinical assessment}

Interviews included the Mini international Neuropsychiatric Interview for the assessment of DSM-IV Axis I psychiatric diagnoses ${ }^{11}$ and the Scale of Suicidal Ideation ${ }^{12}$ for the assessment of suicidal ideation in the past 7 days, administered by a masters or doctoral level psychologist. For veterans with a history of a suicide attempt, the Columbia Suicide History Form ${ }^{13}$ and the Suicide Intent Scale ${ }^{14}$ were administered to characterize intention to die during the most recent attempt and lethality of all attempts. All suicide history forms were discussed in consensus, including ratings of lethality. Clinicians also collected basic demographic information, including use of current medications, and reviewed the electronic medical chart for confirmation of medication use and diagnosis. Trauma history was obtained using the Childhood Trauma Questionnaire. ${ }^{15}$

The process used to create comparable groups of suicide attempters and non-attempters consisted of first selecting individuals who had made the most recent and lethal attempts and then identifying a comparable control based on race/ethnicity, gender and age (within 5 years). An additional consideration included psychiatric diagnosis and medication status, resulting in 20 pairs. Data from one individual were excluded following gene expression assay because the sample showed signs of poor quality and DNA contamination in the reverse transcriptase polymerase chain reaction (RT-PCR) validation, resulting in a final sample size of 39.

\section{Blood drawing and processing}

Forty milliliters of blood were drawn via venipuncture between 0900 and 1000 hours, and collected into PAXgene RNA tubes, centrifuged immediately and stored for further extraction of RNA at the Integrative Systems Biology Lab at the US Army Center for Environmental Health Research. Total RNA was isolated using PAXgene Blood miRNA kit according to the manufacturer's protocol (Qiagen, Hilden, Germany). RNA quality and quantity were determined using NanoDrop 2000 spectrometer (Thermo Fisher, Wilmington, DE, USA) and Agilent 2100 Bioanalyzer (Agilent Technologies, Santa Clara, CA, USA).

\section{mRNA array hybridization and image processing}

Whole-Human Genome Microarray 60 mer Oligo slides printed with Agilent SurePrint Technology were obtained from Agilent Technologies (Agilent Technologies). The slides contained $\sim 41000$ genes and transcripts. Agilent Low Input Quick Amp Labeling Kit, along with Agilent TwoColor Spike-Mix (Agilent Technologies) was used to label experimental (Cy5-labeled) and reference samples-Stratagene Human universal reference RNA (Cy3-labeled, Agilent Technologies). All labeled sample reactions were then subjected to fragmentation followed by $17 \mathrm{~h}$ hybridization using the Agilent Gene Expression Hybridization Kit.

\section{mRNA data acquisition and analysis}

Following scanning and extraction, individual mRNA microarrays were LOESS-normalized ${ }^{16}$ and the bottom $30 \%$ of the distribution of probes were filtered out ${ }^{17}$ as were those that did not have a corresponding RefSeq gene annotation. Quality-control examination of arrays, normalization and primary identification of differentially regulated molecules were performed using custom R scripts (R Development Core Team) with Bioconducter library packages. ${ }^{18}$ No outlier samples or technical batch effects were identified.

\section{Microarray validation}

Twenty-nine mRNA probes with the lowest $P$-values were selected for further validation using RT-PCR. In addition to the sample that was removed because of low signal and DNA contamination, seven samples showed issues with RT-PCR internal controls and were removed for validation purposes. In re-analyzing mRNA microarray values on the remaining 32 individuals, 27 of the 29 probes retained a $P$-value below 0.05 . Four probes showed attempter versus control differences in the RTPCR data with a $P$-value below 0.05 , all in directional agreement with the microarray results. Overall, 18 of the 29 probes moved in the same direction between microarray and RT-PCR.

\section{Statistical and pathway analyses}

$t$-tests and $X^{2}$-analyses were used to evaluate group differences between attempters and controls on demographic and clinical variables. Principal component (PC) analyses were conducted on gene expression data to identify outlier samples and any clinical or sample-processing factors (that is, confounds) that were associated with global gene expression patterns. Moderated $t$-test with a Benjamini and Hochberg false discovery rate correction was used to quantify individual gene differences between attempters and controls. IPA (QIAGEN Redwood City, www.qiagen.com/ ingenuity), which uses open source databases and proprietary hand curation to annotate gene functions, was used for primary pathway analysis for differentially regulated mRNA between attempters and nonattempters and to identify activated or suppressed upstream regulators. Cytoscape, ${ }^{19}$ with networkanalyst ${ }^{20}$ visualization, incorporating data from Kyoto Encyclopedia of Genes and Genomes (KEGG), ${ }^{21}$ wikipathways ${ }^{22}$ and reactome, ${ }^{23}$ was used to find alternative connections and annotation enrichment between differentially expressed genes.

\section{RESULTS}

Table 1 summarizes demographic and clinical characteristics of veterans who had a history of suicide attempt(s) compared to psychiatric controls to further demonstrate the comparability of the samples. As expected, the groups did not differ by age, gender, race or ethnicity. The two groups reported comparable levels of childhood trauma exposure and had similar rates of mood disorder, PTSD and alcohol-use disorder. Veterans who had attempted suicide were also more likely to have a substance use disorder, reflected by a higher rate of narcotic or marijuana dependence. The groups were similar with respect to use of psychotropic medications or use of any type of medication.

PC analyses of mRNA probes revealed a strong separation of attempters from non-attempters in the first two PC. These two components accounted for 34.8 and $16.8 \%$ of the variance in the gene expression data, respectively, and the top five PCs accounted for $68 \%$ of total variance. To investigate the influence of possible confounding clinical variables on mRNA expression levels, clinical variables and demographic variables with at least a $10 \%$ frequency were correlated with loadings for the first two PC dimensions. Suicide attempter status was most strongly correlated with the first two components, whereas gender was correlated with the second component. Other potential confounds including age, use of medication for Type II diabetes, PTSD, bipolar disorder and major depressive disorder were not strongly associated with the major PCs of the mRNA expression data.

Given the limited sample size and low power to detect differences, no individual gene expression probes emerged with a false discovery rate-corrected $P$-value below 0.05 . For the purposes of conducting further downstream pathway enrichment and network analysis, 853 probes that had absolute fold change greater than 0.5 and an uncorrected $P$-value below 0.01 were considered to represent differentially expressed probes. This 
Table 1. Demographic and clinical characteristics in veterans without $(-)$ or with $(+)$ a history of suicide attempts

\begin{tabular}{lrrr}
\hline & Suicide history $-(\mathrm{n}=21)$ & Suicide history+ $(\mathrm{n}=18)$ & Test statistic \\
\hline Age & $45.19(2.94)$ & $45.33(3.17)$ & $\mathrm{F}(1,37)=0.001, P=\mathrm{ns}$ \\
Men \# (\%) & $18(85.7)$ & $16(88.9)$ & $X^{2}(1)=0.087, P=\mathrm{ns}$ \\
Black \# (\%) & $7(33.3)$ & $9(50.0)$ & $X^{2}(2)=3.24, P=\mathrm{ns}$ \\
Hispanic \# (\%) & $9(42.9)$ & $6(33.3)$ & $X^{2}(1)=0.37, P=\mathrm{ns}$ \\
Childhood Trauma Questionnaire-mean (s.d.) & $9.05(0.68)$ & $11.59(1.12)$ & $U=114,\left(n_{1}=19 n_{2}=18\right), P=\mathrm{ns}$ \\
Mood disorder \# (\%) & $16(76.2)$ & $16(88.9)$ & $7(38.9)$ \\
Posttraumatic stress disorder \# (\%) & $7(33.3)$ & $3(16.7)$ & $X^{2}(1)=1.06, P=\mathrm{ns}$ \\
Current alcohol use disorder \# (\%) & $2(9.5)$ & $15(83.3)$ & $X^{2}(1)=0.13, P=\mathrm{ns}$ \\
Lifetime alcohol use disorder \# (\%) & $13(61.9)$ & $10(55.6)$ & $X^{2}(1)=0.44, P=\mathrm{ns}$ \\
Current substance-use disorder \# (\%) & $3(14.3)$ & $15(83.3)$ & $X^{2}(1)=2.20, P=\mathrm{ns}$ \\
Lifetime substance-use disorder \# (\%) & $7(33.3)$ & $14(77.8)$ & $X^{2}(1)=7.43, P=0.006$ \\
Current medication use \# (\%) & $18(85.7)$ & $14(77.8)$ & $X^{2}(1)=9.85, P=0.002$ \\
Current psychotropic use \# (\%) & $13(61.9)$ & $X^{2}(1)=0.42, P=\mathrm{ns}$ & $X^{2}(1)=0.28, P=\mathrm{ns}$ \\
\hline
\end{tabular}

Abbreviations: CTQ, Childhood Trauma Questionnaire; ns, not significant. $\mathrm{p}=\mathrm{ns}$, no significant difference at $P \leqslant 0.05$. ${ }^{\mathrm{a}}$ Two people self-identified as bi-racial . ${ }^{\mathrm{b}}$ Two people did not complete the CTQ. A non-parametric test was used because of a skewed distribution.

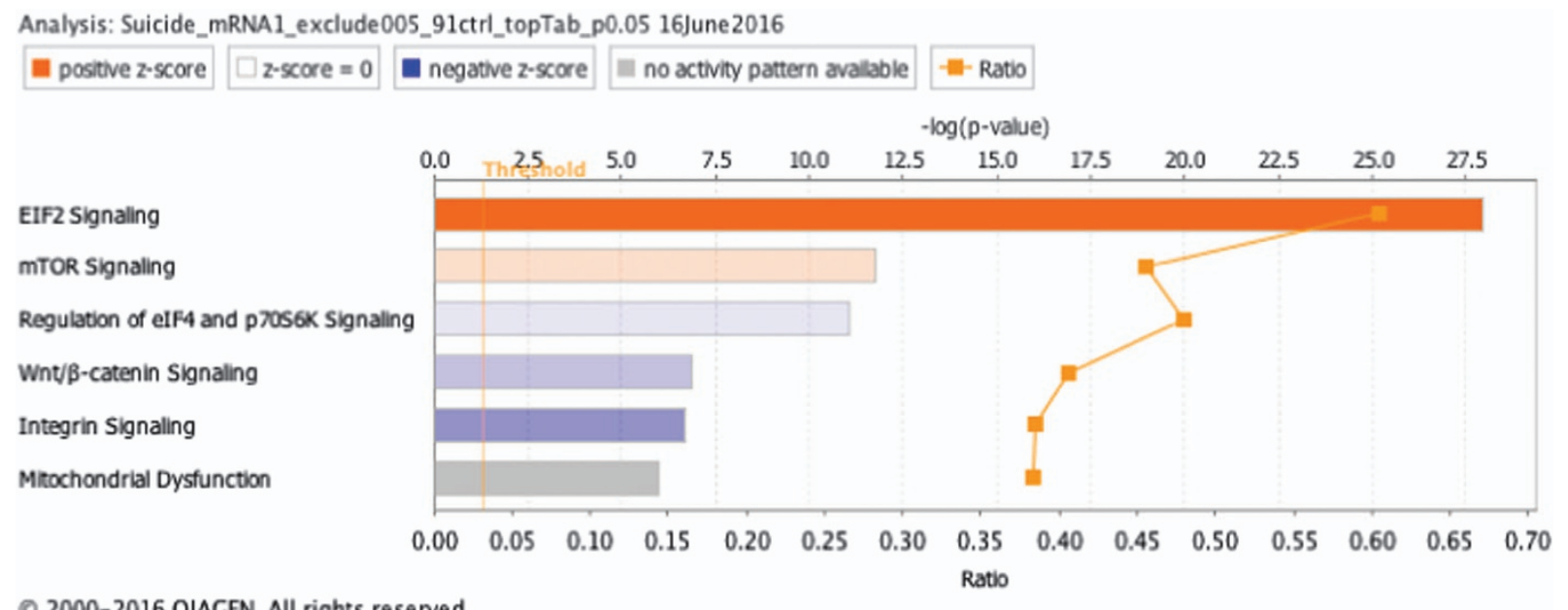

Figure 1. Top 5 enriched IPA canonical pathways $(P<0.01)$ using differentially expressed genes $(P<0.05$, absolute log2fc $>0.5)$. The copyright refers to the software program. The figure has not been published previously. IPA, Ingenuity Pathway Analysis.

cutoff $P$-value corresponded to a false discovery rate of $11.9 \%$. Lower gene expression was more common in veterans who had attempted suicide relative to those who had not (that is, 607 of the 853 probes). Higher gene expression in attempters was observed in 246 probes.

Several highly enriched canonical pathways were identified with IPA including eukaryotic initiation factor (eIF) 2 signaling, mammalian target of rapamycin (mTOR) signaling and regulation of elF4 and p70S6K signaling (Figure 1). Three additional pathways, including Wnt/ $\beta$-catenin Signaling, Integrin Signaling and mitochondrial dysfunction, were less highly enriched, but still had $P$-values $<10^{-5}$. A network analysis of genes associated with the three most highly enriched pathways noted above showed that several genes associated with the mTOR pathway were highly connected downregulated genes, including AKT1, AKTS1, TSC2 and elF4G1. In contrast, upregulated genes included a large number of ribosomal proteins and elF family members (see Figure 2). Additional analyses in Cytoscape using reactome, KEGG and wikipathway annotations also identified the WNT signaling pathway as well as Focal Adhesion, Oxidative Phosphorylation, mRNA processing and Ribosomal Proteins.

Gene expression in enriched pathways. Gene expression magnitude and significance for the top 50 probes representing individual genes within the elF2 signaling pathway that distinguished attempters from non-attempters showed that many were shared between the elF4 and eIF2 pathways (see Table 2). While the majority of top probes were downregulated (33 and 37 out of 50 , respectively, for elF2 and elF4), most elF family genes were upregulated. Downregulated probes in these pathways represent bone morphogenetic proteins and several growth factors, including heparin binding growth factor, connective tissue growth factor and several vascular endothelial growth factor and platelet derived growth factor family members.

Gene expression magnitude and significance for the top 50 probes representing individual genes within the mTOR pathway showed that 12 of the top 50 probes were more highly expressed in attempters. The DVL1 gene was represented by both significantly up- and downregulated probes. The mTOR KEGG pathway showed a pattern of downregulation for genes in and around mTOR complex 1 and, including mTOR itself. RICTOR in the mTOR complex 2 and several downstream elF genes were upregulated. Closer examination of the genes that were downstream of mTOR showed that most were moving in the direction predicted by mTOR suppression (see Figure 3).

With respect to the WNT signaling pathway, six separate members of the proteasome subunit gene family were 


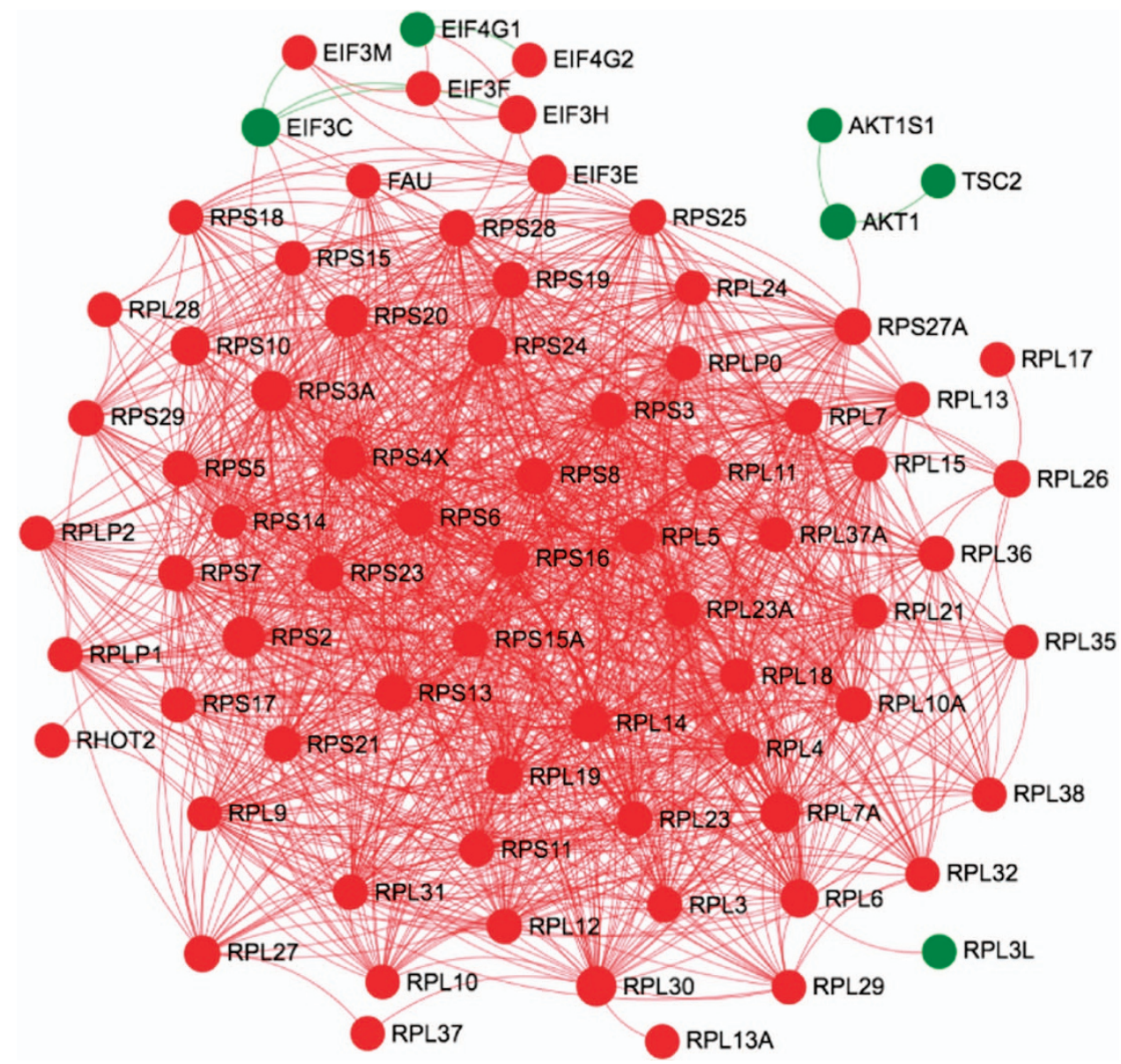

Figure 2. The corresponding networks for the top three canonical pathways. Pathway generated in Cytoscape using Fisher exact test at FDR (q) $<0.05$ and visualized using Networkanalyst. Genes upregulated in veterans with a history of suicide attempt relative to veterans without a history of attempt are shown in red and downregulated genes are shown in green. FDR, false discovery rate.

upregulated (at $P<0.012$ ). Four cadherin $(\mathrm{CHD})$, four g-protein (GN) and two LRP gene family members were downregulated in this pathway. Of the 280 probes representing genes identified with IPA in the elF2 pathway, $31(11 \%)$ had a $P$-value less than 0.01 . Of these 31 probes, only seven showed higher gene expression in attempters, and five of these genes represent elF3 and elF4 family members. Downregulated probes in this pathway represent bone morphogenetic protein and several growth factors including VDGFB, heparin binding growth factor, connective tissue growth factor and platelet derived growth factor family members.

\section{DISCUSSION}

Results of Ingenuity Pathway Analyses identified several pathways of interest that may be associated with suicide attempts, adding to a growing body of research that examines molecular markers of suicidal behavior in blood. Some of these pathways, or specific genes in the pathways, have been linked to suicide in prior studies of both blood and brain tissue. A review of the differentially expressed gene lists from three recently published studies that examined gene expression in blood ${ }^{3-5}$ shows overlap with some of the differentially expressed genes (DEGs) observed in the current study. In addition, examination of the common DEGs shows that the direction of gene expression (for example, upregulated versus downregulated in association with suicide) was nearly always the same. For example, the TNFSF1 (Tumor
Necrosis Factor (Ligand) Superfamily, Member 10) gene was upregulated in attempters versus non-attempters, an association that was also observed by Le-Niculescu et al..$^{3}$ in men reporting a high level of suicidal ideation. Kim et al. ${ }^{24}$ also reported that this gene was overexpressed in prefrontal cortex post-mortem tissue in people with schizophrenia who had died from suicide. This gene codes for a cytokine that is part of the tumor necrosis factor family. In addition, the PIK3C3 (Phosphatidylinositol 3-Kinase Catalytic Subunit Type 3) was upregulated in suicide attempters, consistent with results reported by Levey et al. $^{4}$ in a sample of women with high suicidal ideation. A single-nucleotide polymorphism in this gene was also associated with antidepressant treatment-emergent suicidal ideation in a genome wide association study of depressed inpatients. ${ }^{25}$ These results provide partial replication of gene expression associations with suicidal behavior, extending the research in civilian samples to US veterans.

It is also of great functional value to identify pathways or consider how gene families are regulated together in association with suicide. IPA identified the elF2 pathway as a highly enriched canonical pathway associated with suicide attempter status. Of note, over $10 \%$ of the probes representing the genes of this pathway were differentially expressed in attempters versus nonattempters. Moreover, several members of the elF3 and elF 4 gene families were significantly upregulated in suicide attempters (also represented in Table 2). Proteins coded by these genes form the mammalian ribosome initiation complex at the $5^{\prime}$ end of an mRNA 
Table 2. Gene expression significance (uncorrected $P$-value) and magnitude (fold change for suicide attempters versus controls) for the top 50 probes representing individual genes within the (a) EIF2, (b) mTOR, (c) EIF4 and (d) WNT signaling pathways.

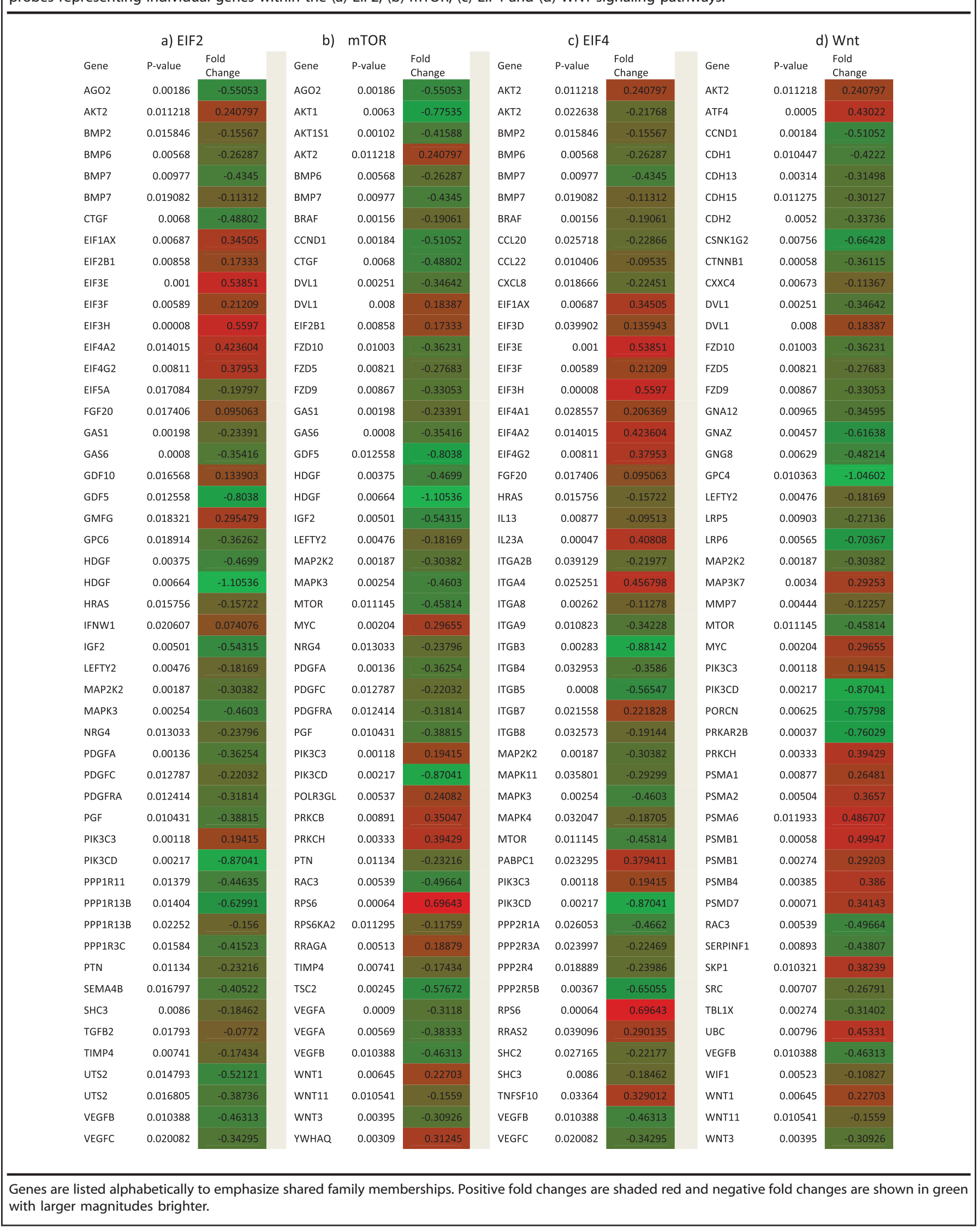




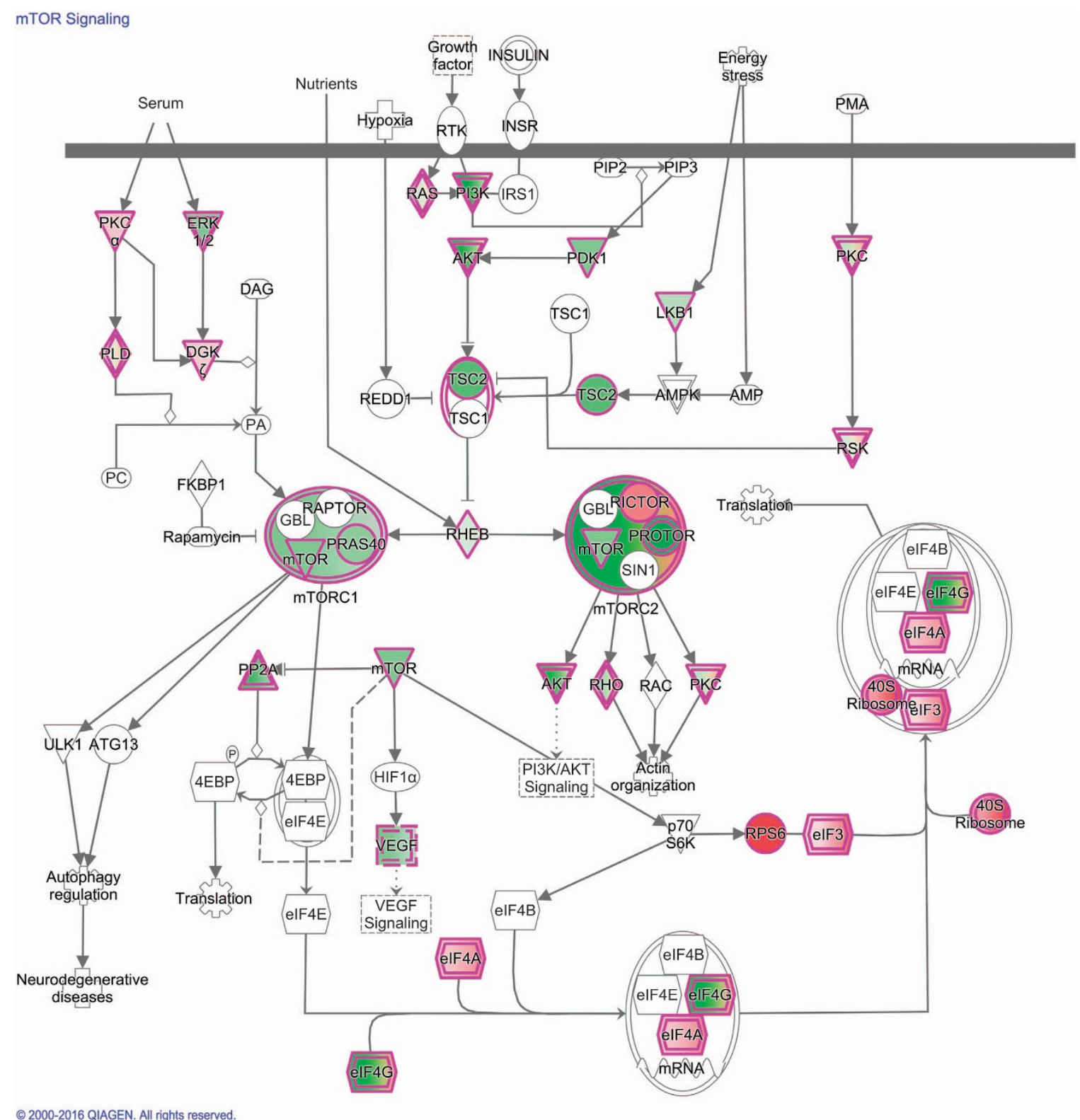

Figure 3. mTOR KEGG pathway colored by log fold change values for genes expressed at absolute log2fc $>0.5$ and $P<0.05$ (red upregulated and green downregulated). The copyright refers to the software program. The figure has not been published previously. KEGG, Kyoto Encyclopedia of Genes and Genomes; mTOR, mammalian target of rapamycin.

(reviewed in Hinnebusch and Lorsch ${ }^{26}$ ), which is regulated by mTOR, another pathway of interest identified in the current study. Although elF2 and elF4 pathways have not been linked to suicide in prior work, gene expression level of elF2 (and mTOR and WNT) was downregulated in one published report examining postmortem tissue in people who had a schizophrenia diagnosis relative to non-psychiatric controls. ${ }^{27}$

The mTOR pathway also emerged as an important pathway in the present study, consistent with prior suicide research. Niculescu et al. ${ }^{5}$ reported that KEGG analyses identified the mTOR pathway as the top pathway associated with suicide in a sample of men with mood and psychotic disorders. As this pathway has been identified in analyses of both blood and brain tissue, this suggests that it may be important for prediction both suicide attempts and completion. The mTOR pathway has been linked to broad constructs important for psychiatric conditions including learning and memory and brain development. ${ }^{28,29}$ With respect to more specific diagnoses, Jernigan et $a .^{30}$ reported that protein expression for mTOR and related genes in the pathway (p70S6K, elF4B and p-elF4B) were lower in post-mortem tissue of people with depression compared with psychiatric controls. The mTOR pathway has also been implicated in the action of ketamine, a fastacting antidepressant that has been shown to reduce suicidal ideation in open label and clinical trials. ${ }^{31}$ In rats, ketamine administration is associated with an increase in synaptic signaling proteins in the mTOR pathway and altered behavior in putative depressogenic paradigms. ${ }^{32}$ Blockade of mTOR signaling with rapamycin completely blocked ketamine-induced synaptogenesis and alterations in behavior. ${ }^{32}$

The WNT pathway was also identified as relevant for suicide in the current analyses. This pathway is also critical for neuronal development ${ }^{33}$ and has been implicated in prior molecular studies of suicide. For example, Ren et al. ${ }^{34}$ examined protein and gene expression of GSK-3 $\beta$ and $\beta$-catenin, two genes in the WNT pathway and reported that protein and gene expression of both genes was lower in prefrontal cortex tissue of adolescents who 
died from suicide, relative to control participants who died from other causes. Similarly, in another study of prefrontal cortex tissue, protein levels of $\beta$-catenin and pGSK3 $\beta$ were lower in people who died from suicide, regardless of mood disorder diagnoses. ${ }^{35}$ Willour et al. ${ }^{36}$ also identified a region on Chromosome 2 (2p25) that was associated with suicide attempts in a sample of patients with biopolar disorder. The single-nucleotide polymorphisms in this region are in a linkage disequilibrium block that includes the ACP1 (acid phosphatase 1) gene, which influences WNT signaling. Follow-up analyses in an independent sample (post-mortem tissue) showed that $A C P 1$ gene expression was higher in people with bipolar disorder who had completed suicide. Finally, WNT signaling is also regulated by lithium, which has long been used to prevent suicidal behavior in people with bipolar disorder and other psychiatric diagnoses. ${ }^{37}$

Limitations of the results reported here must be acknowledged. First, the sample size was small and not sufficiently powered to draw conclusions for individual genes. Second, the DEG significance cutoffs were lowered for pathway enrichment analysis. This introduces the danger of adding noise rather than signal at the pathway level, but the top multiple comparison-corrected pathway-level $P$-values remained highly significant. Moreover, as noted above, the results reported here are in accord with previously published results in studies that incorporated blood and postmortem samples. Third, rather than examining gene expression in the larger sample of veterans who were phenotyped, we opted to match groups based on race, age and psychiatric background. The increase in efficiency of the design with the ability to rule out potential confounding influences was thought to outweigh any loss of statistical power. The results of the PCA suggest that many of the other potential confounds (for example, medication use) were not strongly associated with the major PCs of the mRNA expression data. In particular, we note that linear regression analyses showed that when attempter status and current substance-use disorder were included in the same model, only the attempter status variable was significantly associated with PC1, $t=2.36, P=0.0238$. Current substance use $t=0.70, P=0.49$. Similar results were observed when attempter status and lifetime substance-use disorder were included in the same model for PC1, attempter status $t=2.47, P=0.018$; lifetime substance use $t=0.368, P=0.71$. The results of these analyses suggest that substance-use diagnoses do not account for the association between gene expression and suicide history. However, we acknowledge that the relatively low frequency of some of these clinical and demographic variables is associated with reduced power to observe a significant relationship. Fourth, it should be acknowledged that the group of patients who did not report a history of suicide attempts in the past may make an attempt in the future, which highlights the importance of conducting longitudinal research. Finally, with respect to methodology, blood samples were drawn into PAXgene tubes, which raised the question of whether white blood cell count could explain group differences in results. Future studies in well-defined subpopulations of blood cells should be conducted.

In summary, gene expression analyses conducted in a small study of veterans with diverse psychiatric diagnoses identified three pathways of interest that may be associated with suicide attempts. The mTOR and WNT signaling pathways have also been implicated in treatments for suicide, suggesting further research on these pathways may lead to newer or more improved means of treating suicidal ideation and behavior. Notably, the mTOR and WNT pathways are associated with suicide completion, and their prominence in our results using peripheral tissue suggests that the pathways may represent treatment targets and or trait markers of risk for suicide attempts and completion. The results reported here represent an extension of previous work in several important ways. First, to our knowledge, this is the first sample reported in the literature that included only US veterans. Second, the psychiatric profile of the research participants was diverse, including people with PTSD and substance-use disorders. This enhances the clinical utility of the findings and enabled a comparison of suicide attempts rather than background psychiatric symptomatology. To conclude, the overall high level of pathway and gene-level agreement between this study and prior work demonstrate that the major molecular signatures of suicide attempts in veterans are shared with other populations.

\section{CONFLICT OF INTEREST}

The authors declare no conflict of interest.

\section{ACKNOWLEDGMENTS}

The work reported here was supported by a grant from the American Foundation for Suicide Prevention.

\section{DISCLAIMER}

The views, opinions and/or findings contained in this report are those of the author(s) and should not be construed as official Department of the Army position, policy or decision, unless so designated by other official documentation. Citations of commercial organizations or trade names in this report do not constitute an official Department of the Army endorsement or approval of the products or services of these organizations.

\section{REFERENCES}

1 Prevention USDoVAOoS (2016). Suicide among veterans and other Americans. Available at http://www.mentalhealth.va.gov/docs/2016suicidedatareport.

2 Turecki G. The molecular bases of the suicidal brain. Nat Rev Neurosci 2014; 15: 802-816.

3 Le-Niculescu H, Levey DF, Ayalew M, Palmer L, Gavrin LM, Jain N et al. Discovery and validation of blood biomarkers for suicidality. Mol Psychiatry 2013; 18: $1249-1264$.

4 Levey DF, Niculescu EM, Le-Niculescu H, Dainton HL, Phalen PL, Ladd TB et al. Towards understanding and predicting suicidality in women: biomarkers and clinical risk assessment. Mol Psychiatry 2016; 21: 768-785.

5 Niculescu AB, Levey DF, Phalen PL, Le-Niculescu H, Dainton HD, Jain $\mathrm{N}$ et al. Understanding and predicting suicidality using a combined genomic and clinical risk assessment approach. Mol Psychiatry 2015; 20: 1266-1285.

6 Mullins N, Hodgson K, Tansey KE, Perroud N, Maier W, Mors O et al. Investigation of blood mRNA biomarkers for suicidality in an independent sample. Transl Psychiatry 2014; 4: e474.

7 Kemp J, Bossarte RM. Surveillance of suicide and suicide attempts among veterans: addressing a national imperative. Am J Public Health 2012; 102(Suppl 1): e4-e5.

8 Nock MK, Stein MB, Heeringa SG, Ursano RJ, Colpe L, Fullerton CS et al. Prevalence and correlates of suicidal behavior among soldiers: results from the Army Study to Assess Risk and Resilience in Servicemembers (Army STARRS). JAMA Psychiatry 2014; 71: 514-522.

9 LeardMann CA, Powell TM, Smith TC, Bell MR, Smith B, Boyko EJ et al. Risk factors associated with suicide in current and former US military personnel. JAMA 2013; 310: 496-506.

10 Mann JJ, Waternaux C, Haas GL, Malone KM. Toward a clinical model of suicidal behavior in psychiatric patients. Am J Psychiatry 1999; 156: 181-189.

11 Sheehan DV, Lecrubier Y, Harnett-Sheehan K, Amorim P, Janavs J, Weiller E et al. The M.I.N.I. International Neuropsychiatric Interview (M.I.N.I.). The development and validation of a structured diagnostic psychiatric interview . J Clin Psychiatry 1998; 59(suppl 20): 22-33.

12 Beck AT, Kovacs M, Weissman A. Assessment of suicidal intention: the scale for suicide ideation. J Consult Clin Psychol 1974; 47: 343-352.

13 Oquendo MA, Halberstam B, Mann JJ. Risk factors for suicidal behavior: utility and limitations of research instruments. In: First $M($ ed). Standardized Evaluation in Clinical Practice. American Psychiatric Press: Washington, DC, USA, 2003, 103-130.

14 Beck AT, Schuyler D, Herman I. Development of suicidal intent scales. In: Beck AT, Resnik HLP, Lettieri DJ (eds). The Prediction of Suicide. Charles Press: Bowie, MD, USA, 1974, 45-56.

15 Bernstein DP, Fink L. Childhood Trauma Questionnaire: a Retrospective Report Manual. The Psychological Corporation: San Antonio, TX, USA, 1998. 
16 Smyth GK, Speed T. Normalization of cDNA microarray data. Methods 2003; 31: 265-273.

17 Hackstadt AJ, Hess AM. Filtering for increased power for microarray data analysis. BMC Bioinformatics 2009; 10: 11.

18 Gentleman RC, Carey VJ, Bates DM, Bolstad B, Dettling M, Dudoit S et al. Bioconductor: open software development for computational biology and bioinformatics. Genome Biol 2004; 5: R80.

19 Shannon P, Markiel A, Ozier O, Baliga NS, Wang JT, Ramage D et al. Cytoscape: a software environment for integrated models of biomolecular interaction networks. Genome Res 2003; 13: 2498-2504.

20 Xia J, Gill EE, Hancock RE. NetworkAnalyst for statistical, visual and network-based meta-analysis of gene expression data. Nat Protoc 2015; 10: 823-844.

21 Kanehisa M, Sato Y, Kawashima M, Furumichi M, Tanabe M. KEGG as a reference resource for gene and protein annotation. Nucleic Acids Res 2016; 44: D457-D462.

22 Kelder T, van lersel MP, Hanspers K, Kutmon M, Conklin BR, Evelo CT et al. WikiPathways: building research communities on biological pathways. Nucleic Acids Res 2012; 40(Database issue) D1301-D1307.

23 Milacic M, Haw R, Rothfels K, Wu G, Croft D, Hermjakob H et al. Annotating cancer variants and anti-cancer therapeutics in reactome. Cancers 2012; 4: 1180-1211.

24 Kim S, Choi KH, Baykiz AF, Gershenfeld HK. Suicide candidate genes associated with bipolar disorder and schizophrenia: an exploratory gene expression profiling analysis of post-mortem prefrontal cortex. BMC Genomics 2007; 8: 413.

25 Menke A, Domschke K, Czamara D, Klengel T, Hennings J, Lucae S et al. Genomewide association study of antidepressant treatment-emergent suicidal ideation. Neuropsychopharmacology 2012; 37: 797-807.

26 Hinnebusch AG, Lorsch JR. The mechanism of eukaryotic translation initiation: new insights and challenges. Cold Spring Harb Perspect Biol 2012; 4.

$27 \mathrm{Chu}$ T, Liu Y, Kemether E. Thalamic transcriptome screening in three psychiatric states. J Hum Genet 2009; 54: 665-675.

28 Graber TE, McCamphill PK, Sossin WS. A recollection of mTOR signaling in learning and memory. Learn Memory 2013; 20: 518-530.

29 Lee DY. Roles of mTOR signaling in brain development. Exp Neurobiol 2015; 24: 177-185.

30 Jernigan CS, Goswami DB, Austin MC, lyo AH, Chandran A, Stockmeier CA et al. The mTOR signaling pathway in the prefrontal cortex is compromised in major depressive disorder. Progr Neuropsychopharmacol Biol Psychiatry 2011; 35: 1774-1779.

31 Naughton M, Clarke G, O'Leary OF, Cryan JF, Dinan TG. A review of ketamine in affective disorders: current evidence of clinical efficacy, limitations of use and preclinical evidence on proposed mechanisms of action. J Affect Disord 2014; 156: 24-35.

32 Li N, Lee B, Liu RJ, Banasr M, Dwyer JM, Iwata $M$ et al. mTOR-dependent synapse formation underlies the rapid antidepressant effects of NMDA antagonists. Science 2010; 329: 959-964.

33 Meffre D, Grenier J, Bernard S, Courtin F, Dudev T, Shackleford G et al. Wnt and lithium: a common destiny in the therapy of nervous system pathologies? Cell Mol Life Sci 2014; 71: 1123-1148.

34 Ren X, Rizavi HS, Khan MA, Dwivedi Y, Pandey GN. Altered Wnt signalling in the teenage suicide brain: focus on glycogen synthase kinase-3beta and beta-catenin. Int J Neuropsychopharmacol 2013; 16: 945-955.

35 Karege F, Perroud N, Burkhardt S, Fernandez R, Ballmann E, La Harpe R et al. Protein levels of beta-catenin and activation state of glycogen synthase kinase3beta in major depression. A study with postmortem prefrontal cortex. J Affect Disord 2012; 136: 185-188.

36 Willour VL, Seifuddin F, Mahon PB, Jancic D, Pirooznia M, Steele J et al. A genomewide association study of attempted suicide. Mol Psychiatry 2012; 17: 433-444.

37 Lewitzka U, Severus E, Bauer R, Ritter P, Muller-Oerlinghausen B, Bauer M. The suicide prevention effect of lithium: more than 20 years of evidence-a narrative review. Int J Bipolar Disord 2015; 3: 32.

(\$) This work is licensed under a Creative Commons AttributionNonCommercial-NoDerivs 4.0 International License. The images or other third party material in this article are included in the article's Creative Commons license, unless indicated otherwise in the credit line; if the material is not included under the Creative Commons license, users will need to obtain permission from the license holder to reproduce the material. To view a copy of this license, visit http:// creativecommons.org/licenses/by-nc-nd/4.0/

(c) The Author(s) 2017 